\title{
Renal stones and urinary infection: a study of antibiotic treatment
}

\author{
R H CHINN， ROSALIND MASKELL，J A MEAD， A POLAK
}

between February 1971 and May 1974. All had a well-documented history of persistent or recurrent urinary tract infection despite intermittent antibacterial treatment. Twenty-one had renal calculi and one had passed a calculus immediately before admission to the study.

\section{Summary}

Twenty-two patients in whom renal calculi and urinary infection were closely associated were studied over two to five years. Four patients had previously had stones surgically removed, and five underwent pyelolithotomy during the course of the study. Urinary infection was treated with an appropriate antibacterial agent, and treatment was followed by long-term prophylaxis, usually with cotrimoxazole. A sterile urine was maintained for long periods in all these patients. In four patients, however, apparent stone growth occurred while the urine was sterile.

On entering the study 21 of the 22 patients complained of symptoms. After treatment 19 of the 20 patients who were still attending were symptom-free. Six of the 22 patients entered the study with raised levels of serum creatinine; levels fell in four and remained raised in two.

This antibacterial regimen, either alone or after surgery, will usually relieve symptoms and may prevent deterioration of renal function.

\section{Introduction}

The problem of the patient with renal calculi, persistent urinary infection, and no metabolic cause for stone formation is well known to urologists, nephrologists, and bacteriologists. Some now believe ${ }^{1}$ that infection is usually the cause rather than the result of stone formation in these patients. Many of the calculi eventually grow to fill the renal pelvis, resulting in loss of tissue due to obstruction and infection. The patients may present with acute pyelonephritis and eventually progress to nephrectomy, or renal failure if the disease is bilateral. One approach to this problem is surgical removal of the stones at the earliest possible stage combined with vigorous attempts to treat infection and prevent subsequent recurrence, which usually leads to further stone formation. In some patients, however, operation may not be possible or advisable, especially if they have had previous kidney operations. It is therefore important to control infection both after surgery and when surgery is not considered advisable. So far as we know long-term chemotherapy in this type of patient has not been specifically studied, and we therefore present our results over five years.

\section{Patients and methods}

Twenty-two patients (two men aged 57 and 64 years, and 20 women aged 21-70 years (mean age 43 years)) were admitted to the study

Southampton University Department of Renal Medicine, Wessex Renal Unit, and Public Health Laboratory, St Mary's General Hospital, Portsmouth

R H CHINN, MRCP, lecturer in medicine

ROSALIND MASKELL, BM, BCH, senior research associate

J A MEAD, MRCGP, clinical assistant

A POLAK, MD, FRCP, professor of renal medicine Four patients had had a pyelolithotomy and three had previously passed stones. All patients were screened for hyperparathyroidism, hypercalciuria, urate nephropathy, cystinuria, and renal tubular acidosis, with negative results.

Urinary tract infection was diagnosed by culturing a carefully collected mid-stream urine specimen on CLED agar using a $0.01-\mathrm{m}$ fused platinum standard loop. Organisms were identified by standard methods, ${ }^{2}$ and strains of Escherichia coli were serotyped by the Salmonella and Shigella Reference Laboratory (Central Public Health Laboratory). Microscopy of the centrifuged deposit was carried out by one of us in the outpatient clinic to prevent any possible inaccuracy caused by refrigerating the specimen before transporting it to the laboratory. Ten high power fields (hpf) were examined: an average of two or more leucocytes or red blood cells per hpf was recorded as pyuria or haematuria respectively. We recognise that this is an insensitive test, especially for haematuria, as normal urine usually contains less than one red cell per $10 \mathrm{hpf}$, and patients who did not have haematuria on our criterion might still have too many red cells in the urine.

Intravenous pyelography was carried out on all patients and micturating cystourethrography on 13 patients. A plain film of the abdomen was taken each year. Stone size was recorded arbitrarily from the $x$-ray films as small (longest diameter $<0.5 \mathrm{cms})$, medium $(0.5-2$ $\mathrm{cms}$ ), and large ( $>2 \mathrm{cms}$ ). Eight patients were admitted for cystoscopy and localisation of infection by ureteric catheterisation. ${ }^{3}$ Serum creatinine was measured by autoanalyser.

On admission to the study all patients were treated with a seven-day course of an appropriate agent, usually co-trimoxazole, in therapeutic doses. One patient was given a seven-day course of intramuscular kanamycin after oral chemotherapy had failed. On completion of the therapeutic course a prophylactic or suppressive low-dose regimen was started. Twenty patients received one tablet of co-trimoxazole each night and nitrofurantoin $50 \mathrm{mg}$ nightly was given to two patients with a history of hypersensitivity to sulphonamide or co-trimoxazole. The low-dose regimen was continued until the mid-stream urine specimen had produced no growth on culture for one year. If infection recurred either during treatment or after stopping it, the recurrence was treated with an appropriate agent for seven days and prophylaxis restarted. In reporting the results, prophylaxis which resulted in a sterile mid-stream specimen is referred to as successful prophylaxis (see Discussion).

All patients were seen every three months and more often if infection persisted or recurred. Urine culture was carried out at each visit and between visits if symptoms recurred. Serum creatinine, haemoglobin, red cell count and indices, and white cell count and differential were measured at least once a year, and more often if there were clinical indications.

\section{Results}

The 22 patients were followed for a total of 933 months (range 23-62 months). One patient died from carcinomatosis after 31 months.

\section{ON ADMISSION}

On admission one patient was symptom-free, 11 had micturition symptoms and loin pain, eight had loin pain only, and two had micturition symptoms only. The infecting organism was Proteus mirabilis in 13 patients, $E$ coli in eight, and Proteus and $E$ coli in one. Seventeen patients had pyuria and 10 had haematuria.

Six patients had a raised serum creatinine concentration (fig 1). Intravenous pyelography showed a normal outline and calcyeal pattern in nine patients; obstructive changes behind the stone in one; a 


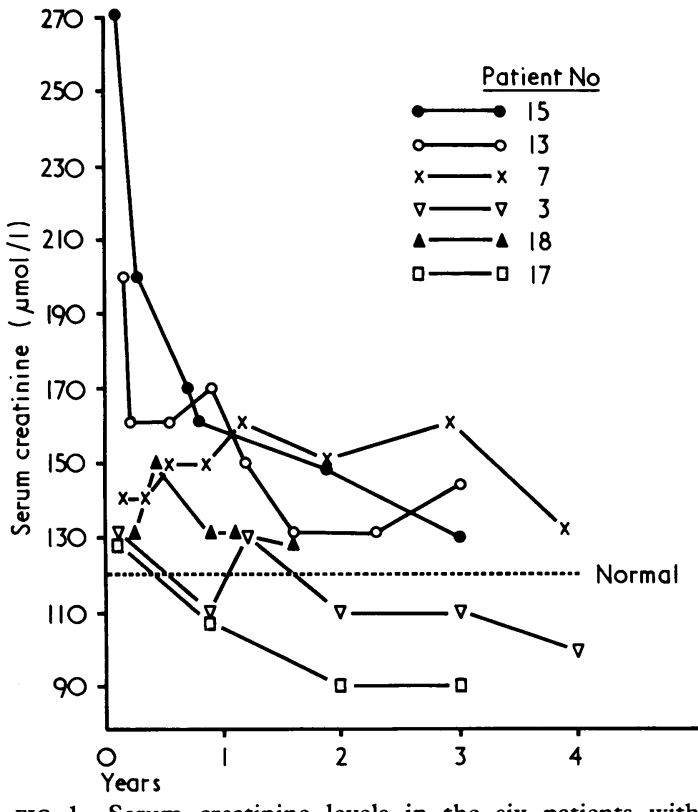

FIG 1 -Serum creatinine levels in the six patients with raised concentrations. The numerical data on which the figure is based are available on request from the authors. Conversion: SI to traditional units-Creatinine: $1 \mu \mathrm{mol} / \mathrm{l}$ $\approx 0.0113 \mathrm{mg} / 100 \mathrm{ml}$

hypertrophied single kidney in one; and pyelonephritic changes in the right kidney in four, in the left kidney in two, and in both kidneys in five.

Seventeen patients had unilateral and four had bilateral stones. Fourteen patients had only one stone; of these three were large, four medium, and seven small. The remaining patients had multiple stones of which two were large, nine medium, and five small. Four patients had had a pyelolithotomy.

Micturating cystourethrography showed no reflux in 12 of the 13 patients examined; one patient had reflux on the same side as her stone.

Ureteric catheterisation showed renal infection in all eight patients examined. Five had infection localised to the kidney containing a

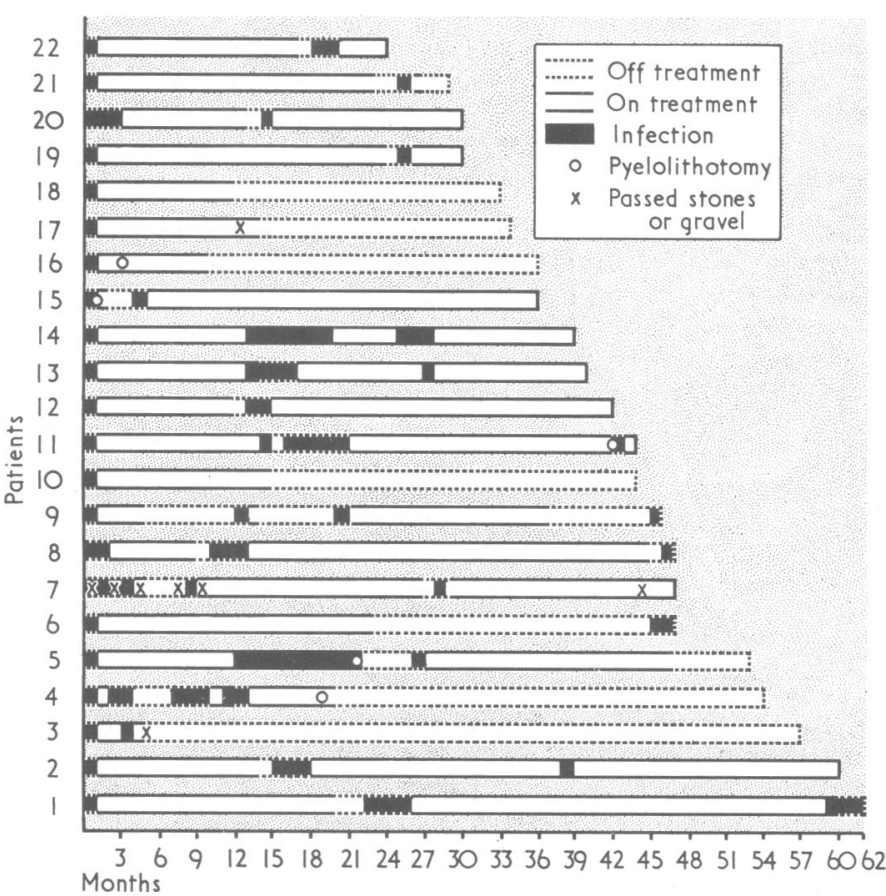

FIG 2-Treatment and recurrence of infection in 22 patients. The numerical data on which the figure is based are available on request from the authors. unilateral stone; two had bilateral infection with unilateral stones (one had bilateral pyelonephritis and the other had had a pyelolithotomy on the other kidney), and one had unilateral infection with bilateral stones.

DURING FOLLOW-UP

Preventing or suppressing infection-Fig 2 shows details of treatment and recurrence of infection. Eighteen patients had 31 recurrences, 10 while on prophylaxis (two of which were with thymine-requiring mutants ${ }^{4}$ ) and 21 off prophylaxis. Fifteen of these occurred within three months of stopping prophylaxis. None of the patients treated with long-term co-trimoxazole experienced any side effects, and no haematological changes occurred.

Symptoms-Seventeen patients became and remained symptom-free while on successful prophylaxis. Of the 20 patients still attending at the time of writing 19 were symptom-free and one, whose urine was sterile, complained of loin pain. Seventeen of the 31 recurrences were not accompanied by symptoms.

Pyuria and haematuria-While on successful prophylaxis 14 out of 22 patients had no pyuria or haematuria as defined above; all had had pyuria and seven had also had haematuria when their urine was infected. Pyuria persisted in three patients, haematuria in three, and pyuria and haematuria in two during successful prophylaxis.

Stones-During the study three patients passed stones or "gravel"; four had a pyelolithotomy; and one had a pyelolithotomy and partial nephrectomy. Infection recurred in three of these five patients after operation and was successfully controlled with prophylaxis. Two patients showed an increase in stone size and two developed apparently new stones during successful prophylaxis.

Serum creatinine-Fig 1 shows the serum creatinine levels of the six patients in whom it was raised above $120 \mu \mathrm{mol} / 1(1.4 \mathrm{mg} / 100 \mathrm{ml})$ initially. The level fell in four of the six, and remained steady in two. It did not rise in any of the other 16 patients.

\section{Discussion}

We have shown in 22 patients in whom renal calculi and urinary infection were closely associated that it is possible to sterilise the urine and keep it sterile for long periods. All but one of the patients had initially complained of symptoms, and all but one became symptom-free. Serum creatinine concentrations did not rise, and they fell in four of the six patients in whom they were originally raised.

Although the urine remained sterile for long periods during antibacterial treatment and prophylaxis, we cannot assume that infection in or around the calculi was eliminated. When renal disease is unilateral the antibiotic is probably cleared predominantly by the unaffected kidney; the bladder urine is sterilised, but organisms may persist in the diseased kidney. That they did so in some of these patients is suggested by the fact that when infection recurred after stopping prophylaxis it usually did so within a short time, indicating relapse rather than reinfection. ${ }^{5}$ Although the same serotype of $E$ coli was usually isolated on recurrence, this is not conclusive proof of relapse, ${ }^{6}$ and we did not type Proteus spp.

Radiologically detectable stone growth occurred in two patients and apparently new stone formation occurred in two others while the urine was sterile. The importance of this finding is difficult to assess. Possibly organisms had persisted in the substance of the stones ${ }^{7}$ and actual stone growth had occurred as a result. Alternatively, the radiological changes may have resulted from calcification of matrix substance already present.

The two principal aims of treatment were to relieve the patients' symptoms and to maintain their renal function. We have shown that chemotherapy, or a combination of surgery and chemotherapy when appropriate, can achieve these aims. Antibacterial treatment must be prolonged, possibly indefinitely in some patients, and until the advent of co-trimoxazole ${ }^{8}$ no satisfactory agent was available for this purpose. Patients who cannot tolerate co-trimoxazole or who develop infection with thymine-requiring mutants ${ }^{4}$ present a problem. Nitrofurantoin is the other antibacterial agent that has been used successfully 
in long-term prophylaxis against urinary infection, ${ }^{9}$ and we used it in two patients. In unilateral disease, however, its very rapid clearance by the normal kidney may result in a poor concentration in the urine excreted by the diseased kidney. When both kidneys are diseased effective urine concentrations may be achieved only in the presence of potentially toxic serum levels. Furthermore, many of these patients have Proteus infection, and nitrofurantoin is usually ineffective against this organism in vivo. We therefore consider co-trimoxazole the agent of choice for use in the way we have described.

The failure of antibiotic treatment to prevent stone growth (or matrix calcification) in four of our patients underlines the need for close collaboration between the physician and the surgeon. The indications for surgery are difficult to define and may be influenced by the degree of supervision of the patient that will be possible. It should be emphasised that four patients in this series, and several others with stones and infection under our care, have had previous stone surgery. Sometimes, therefore, the problem is not whether to operate, but how many times, and this should always be borne in mind before first deciding on surgery. If the presence of infection is an indication for operation it is logical to confirm by a localisation procedure that infection is present in the kidney. This is particularly important, since most patients with stones and urinary infection are women, and there is a possibility therefore that the stones may prove to be fortuitously associated with bacterial cystitis.

We thank Mr J Vinnicombe and Mr G F Abercrombie for surgical advice and co-operation and $\operatorname{Dr} B$ Rowe of the Salmonella and Shigella Reference Laboratory, Colindale, for serotyping the strains of $E$ coli.

\section{References}

${ }^{1}$ Wickham, J E A, British fournal of Urology, 1976, 47, 727.

${ }^{2}$ Cowan, S T, and Steel, $\mathrm{K}$ J, Manual for the Identification of Medical Bacteria. London, Cambridge University Press, 1966.

3 Stamey, T A, Govan, D E, and Palmer, J M, Medicine, 1965, 44, 1.

4 Maskell, R, Okubadejo, O A, and Payne, R H, Lancet, 1976, 1, 834.

5 Grüneberg, R N, fournal of Clinical Pathology, 1970, 23, 259.

${ }^{6}$ Gower, P E, and Tasker, P R W, British Medical fournal, 1976, 1, 684.

${ }^{7}$ Nemoy, N J, and Stamey, T A, fournal of the American Medical Association, 1971, 215, 9.

${ }^{8}$ Cattell, W R, et al, British Medical fournal, 1971, 1, 377.

${ }^{9}$ Bailey, R R, et al, Lancet, 1971, 2, 1112

\title{
Sudden deaths among Finnish conscripts
}

\author{
KIMMO KOSKENVUO
}

British Medical fournal, 1976, 2, 1413-1415

\section{Summary}

The epidemiology of sudden deaths was studied among conscripts in Finland in 1948-72 (660 000 man-years) and among Finnish men aged 15-24 years in 1969-70 (900 000 man-years). The incidence of sudden deaths among the conscripts was 6.8/100 000 man-years. The onset of acute symptoms occurred during strenuous exercise in a third of the conscripts but in only a few of the other young men. Cardiovascular diseases caused two-thirds of the deaths in both groups. Sudden death tended to be more common among conscripts than among other young men during the corresponding period, but overall nonviolent deaths were less common among conscripts than among other young men. On the basis of health records an attempt was made to separate the sudden deaths among conscripts from those among the controls by multiple discriminant analysis. For all practical purposes, however, the separation power proved poor.

\section{Introduction}

Sudden deaths constitute a large proportion of all nonviolent deaths among young men, ${ }^{1-4}$ but I am not aware of any prospective or controlled retrospective study of the epidemiology of sudden deaths among young men. In military service sudden deaths are accurately recorded. The aim of this study was to determine the incidence, causes, and risk factors of non-violent

\footnotetext{
Medical Section, General Headquarters, Defence Forces, 00101 Helsinki 10, Finland

KIMMO KOSKENVUO, MD, chief of medical section
}

sudden death among conscripts in Finland. Moreover, an attempt was made to identify conscripts with a "high" risk of non-violent sudden death.

\section{Subjects and methods}

The study population consisted of nearly 900000 young men aged about 20 years who were Finnish conscripts in 1948-72. Nearly 660000 man-years were covered. A sample of conscripts was used as a control group. One name out of every 500 was drawn from the register of 19-year-olds liable to be drafted in 1950, 1955, 1960, 1965, and 1970 in each of the 25 military districts in Finland. Data on the health of $97 \%$ of these men (418) were available. By the end of 1972 401 of the men had entered and performed military service and they formed the control group of conscripts.

In addition, the sudden deaths of young men aged 15-24 that occurred in 1969-70 in Finland were analysed. About 900000 manyears were covered.

The health data on conscripts who died suddenly, the circumstances and clinical course of the acute illness, and the necropsy findings were analysed. The residence, social class, findings at initial medical examination, and use of medical services during military service were compared in the conscripts who died and their controls. A multiple discriminant analysis was performed with eight variables: $(a)$ the number of days spent as an outpatient during military service, $(b)$ the number of appointments with a doctor during the first 12 weeks of military service, $(c)$ the number of appointments with a doctor during military service, $(d)$ the number of days spent as an inpatient because of an infection during the first 12 weeks of military service, $(e)$ systolic blood pressure at initial examination, $(f)$ weight at initial examination, $(g)$ social class before military service, and $(h)$ the number of appointments with a doctor because of infection during the first 12 weeks of military service.

Finally, those who were conscripts in 1967-72 were compared with the remainder of the Finnish male population aged 15-24 (1969-70). The incidence and cause of sudden death and the possible connection of sudden death with strenuous exercise were the basis for comparison. The following definition of sudden death from disease was used" "When a person not known to have been suffering from dangerous disease, injury, or poisoning is found dead or dies within 24 hours after the onset of the terminal illness, death is said to have been sudden or unexpected." 